\title{
Epigenetic marks in the mature pollen of Quercus suber L. (Fagaceae)
}

\author{
Teresa Ribeiro $\cdot$ Wanda Viegas $\cdot$ Leonor Morais-Cecílio
}

Received: 12 August 2008/Accepted: 13 September 2008/Published online: 30 September 2008

(C) Springer-Verlag 2008

\begin{abstract}
We have analysed the distribution of epigenetic marks for histone modifications at lysine residues $\mathrm{H} 3$ and $\mathrm{H} 4$, and DNA methylation, in the nuclei of mature pollen cells of the Angiosperm tree Quercus suber; a monoecious wind pollinated species with a protandrous system, and a long post-pollination period. The ultrasonic treatment developed for the isolation of pollen nuclei proved to be a fast and reliable method, preventing the interference of cell wall autofluorescence in the in situ immunolabelling assays. In contrast with previous studies on herbaceous species with short progamic phases, our results are consistent with a high level of silent (5-mC and $\mathrm{H} 3 \mathrm{~K} 9 \mathrm{me} 2)$ epigenetic marks on chromatin of the generative nucleus, and the prevalence of active marks (H3K9me3 and H4Kac) in the vegetative nucleus. The findings are discussed in terms of the pollination/fertilization timing strategy adopted by this plant species.
\end{abstract}

Keywords Epigenetic marks - Vegetative nucleus · Generative nucleus $\cdot$ Fagaceae $\cdot$ Delayed fertilization

Communicated by Thomas Dresselhaus.

T. Ribeiro $(\bowtie) \cdot$ W. Viegas · L. Morais-Cecílio

Departamento de Botânica e Engenharia Biológica, Centro de Botânica Aplicada à Agricultura (CBAA), Instituto Superior de Agronomia, Technical University of Lisbon, Tapada da Ajuda, 1349-017 Lisbon, Portugal e-mail: atribeiro@isa.utl.pt

L. Morais-Cecílio

e-mail: 1morais@isa.utl.pt

\section{Introduction}

Chemical modifications of histones and DNA methylation patterns play an important role in regulating chromatin dynamics and transcription. The male germ cells of higher plants are initiated from haploid microspores following an asymmetric division. The nucleus of the microspore cell then migrates to one of the poles, where it undergoes a mitotic division, giving rise to two structurally and functionally different cells. The smaller and elongated cell closest to the pole, generative cell, has a reduced volume of cytoplasm containing relatively few organelles, and is predestined to divide and form the two male sperm nuclei. The larger and spherical cell vegetative cell contains higher amounts of stored molecules such as starch and lipids, and has an important role in pollen grain maturation and germination, and also in pollen tube elongation. Once formed, the generative cell rapidly detaches from the pole and floats freely within the cytoplasm of the vegetative cell, resulting in a unique "cell-within-a-cell" structure in the pollen grain.

Besides different sizes and composition, the generative and vegetative cells also present distinct nuclear configurations. Soon after the asymmetric division of the microspore, the generative nucleus enters the $\mathrm{S}$ phase, and then passes into $\mathrm{G} 2$ assuming a high chromatin condensation pattern. The vegetative nucleus is arrested at G1, and displays a diffuse chromatin appearance (Bino et al. 1990; Tanaka 1997).

Chromatin structure is not only important for DNA packaging, but also plays an active role in diverse biological processes such as gene expression, DNA replication and repair and chromosome recombination and segregation (Fuchs et al. 2006). Different patterns of chromatin organization are associated with characteristic gene expression profiles and distinctive staining properties. Euchromatin represents decondensed and potentially active chromatin, 
whereas heterochromatin is condensed and largely transcriptionally inert. Heterochromatin can be constitutive, when condensation is permanent throughout the cell cycle in all tissues, or facultative when chromatin compaction is developmentally regulated as a result of cellular differentiation (Craiq 2005). Euchromatin and heterochromatin are also characterized by different epigenetic marks associated with specific posttranslational histone modifications, or with distinct levels of cytosines methylated at the position five of the pyrimidine ring $(5-\mathrm{mC})$. Amongst the plethora of well-documented histone modifications, the acetylation and methylation of selected lysine residues $(\mathrm{K})$ of histones $\mathrm{H} 3$ and $\mathrm{H} 4$ seem to be particularly involved in the regulation of gene transcription (Richards and Elgin 2002; Horn and Peterson 2006). Genes residing in euchromatic domains are usually associated with, the hyperacetylation of several lysine residues on histones $\mathrm{H} 3$ and $\mathrm{H} 4$, high levels of $\mathrm{H} 3$ methylation in $\mathrm{K} 4, \mathrm{~K} 36, \mathrm{~K} 79$, and low levels of DNA methylation. Silent heterochromatic domains are characterized by hypoacetylation of $\mathrm{H} 4$, and histone $\mathrm{H} 3$ dimethylation on $\mathrm{K} 9$ and $\mathrm{K} 27$, histone $\mathrm{H} 4$ methylation on K20, and DNA hypermethylation (Berger 2007). Histone methylation marks are present both on hetero- and euchromatin, being specified by the position and number of methyl groups. In plants, for instance, $\mathrm{H} 3 \mathrm{~K} 4$ methylation is restricted to euchromatin and, $\mathrm{H} 3 \mathrm{~K} 9 \mathrm{me} 1,2$, to heterochromatin (review in Fuchs et al. 2006).

Angiosperms display distinct fertilization processes enabling self- or cross-pollination, which in turn can be related with the period of time from pollination to fertilisation (Weterings and Russell 2004). Studies reporting epigenetic mark patterns in pollen nuclei are scarce, and have been restricted to herbaceous plants with short progamic phases (Oakeley et al. 1997; Janousek et al. 2000, 2002; Okada et al. 2006). Quercus suber L. is a monoecious wind-pollinated tree species with a protandrous system and a long progamic phase, taking about 3 months between pollination and the conclusion of the fertilization process (Boavida et al. 1999). In this work we characterize the distribution of several epigenetic marks in each nucleus of the bicellular mature pollen. The study of epigenetic marks in pollen nuclei of $Q$. suber is compared with those observed in Lilium, in order to disclose potential correlations between different pollination/fertilization processes, as well as chromatin dynamics in pollen grain nuclei.

\section{Materials and methods}

Pollen collection

Catkins containing flowers after anthesis were collected from several trees in Sintra, Portugal, during the flowering season in 2008. Dehiscent anthers of Lilium sp. grown in the greenhouse were collected from full open flowers.

Pollen of both species was isolated by shaking the anthers into a copper sieve. The isolated pollen grains were then kept at room temperature in a sealed vials containing silica gel, to avoid any effects due to humidity until used.

Isolation of pollen nuclei by ultrasonic treatment

Isolation of the nuclei of mature pollen was carried out according to Pan et al. (2004), with modifications. Briefly, isolated pollen grains were resuspended in nuclear isolation buffer for woody plants (WPB) (Loureiro et al. 2007), consisting of $0.2 \mathrm{M}$ Tris $\mathrm{HCl}, 4 \mathrm{mM} \mathrm{MgCl} 2 \cdot 6 \mathrm{H}_{2} \mathrm{O}, 2 \mathrm{mM}$ EDTA $\mathrm{Na}_{2} \cdot 2 \mathrm{H}_{2} \mathrm{O}, 86 \mathrm{mM} \mathrm{NaCl}, 10 \mathrm{mM}$ sodium metabisulfite, $1 \%(\mathrm{w} / \mathrm{v})$ PVP-10, $1 \%(\mathrm{v} / \mathrm{v})$ Triton X-100, at $\mathrm{pH}$ 7.5. The suspension of pollen grains $(0.5 \mathrm{ml}$ of WPB per $0.01 \mathrm{~cm}^{3}$ of pollen grains) was treated on ice with an ultrasonic device (Ultrasonic Processor, model UP $50 \mathrm{H}$, Dr. Hielsher $\mathrm{GmbH}$ ) at $40 \mathrm{~W}$, using 0.8 cycle with $80 \%$ amplitude, for $5 \mathrm{~min}$, or with a low magnitude ( $40 \mathrm{~W}, 0.5$ cycles with $40 \%$ magnitude, for $3 \mathrm{~min}$ ). The treated solution was then filtered through miracloth (Calbiochem) and centrifuged at $500 \mathrm{~g}$ for $5 \mathrm{~min}$. According to the amount of pellet obtained, part of the supernatant was carefully discarded and the pellet resuspended in approximately $100 \mu \mathrm{L}$ of the remaining volume.

\section{Pre-treatment of microscope slides}

For enhanced nuclei adhesion, microscope slides were previously immersed in a freshly prepared solution of $2 \%(\mathrm{v} / \mathrm{v})$ APTES (3-aminopropyltriethoxy-silane, Sigma) in acetone for $10 \mathrm{~s}$, followed by a brief wash in acetone and water, and finally air-dried.

Slide preparation and immunostaining of pollen nuclei

For histone immunoanalysis, nuclei suspension aliquots were mounted on APTES-coated slides, and attached to slides by the dry-ice technique (Schwarzacher and Heslop-Harrison 2000). Fixation was carried out on the slides with $200 \mu \mathrm{L}$ of $4 \%$ (w/v) formaldehyde in $1 \times$ PBS during $20 \mathrm{~min}$ at room temperature, and further washed for $5 \mathrm{~min}$ in $1 \times$ PBS. Immunodetection of modified histones was done according to published methods (Houben et al. 2003). To avoid non-specific antibody binding, slides were incubated for $30 \mathrm{~min}$ in a blocking solution of $8 \%(\mathrm{w} / \mathrm{v})$ BSA, $0,1 \%(\mathrm{v} / \mathrm{v})$ Triton $\mathrm{X}-100$ in PBS at room temperature. After washing in $1 \times \mathrm{PBS}$, slides were incubated for $12 \mathrm{~h}$ at $4{ }^{\circ} \mathrm{C}$ in a humid chamber with primary antibodies in the following dilutions: 1:200 (antiH3K9Ac, Upstate 06-942), 1:500 (anti-H3K9me2, Abcam 
AB 7312, and anti-H4K5,K8,K12,K16Ac, Abcam AB 2380) and 1:1,000 (anti-H3K4me3, Abcam AB 8580) in $1 \times$ PBS supplemented with $1 \%$ BSA. The slides were further washed in $1 \times \mathrm{PBS}$ and incubated in Cy3-conjugated anti-rabbit IgG diluted $1: 100$ in $1 \times \mathrm{PBS}, 1 \% \mathrm{BSA}$ for $1 \mathrm{~h}$ at $37^{\circ} \mathrm{C}$. After final washes in $1 \times \mathrm{PBS}$, slides were mounted in antifade containing DAPI as DNA counterstain.

For cytosine methylation immunoanalyses the suspensions of nuclei were mixed with an equal volume of the fixative 3:1 (v/v) ethanol:glacial acetic acid mixture, placed on ice for $10 \mathrm{~min}$ and centrifuged $5 \mathrm{~min}$ at $500 \mathrm{~g}$. The supernatant was discarded and replaced with fresh $3: 1(\mathrm{v} / \mathrm{v})$ ethanol:glacial acetic acid mixture, and this fixation and centrifugation was repeated. According to the amount of pellet obtained, a volume (approximately $100 \mu \mathrm{L}$ ) of new 3:1 fixative was adjusted and the pellet resuspended. Aliquots were dropped on to APTES-coated slides and allowed to dry. Immunolocalization of 5-methylcytosine residues was performed according to published methods (Castilho et al. 1999). Briefly, slides were blocked for $30 \mathrm{~min}$ in $1 \%(\mathrm{w} / \mathrm{v}) \mathrm{BSA}$ in $1 \times \mathrm{PBS}$ supplemented with $0.5 \%(\mathrm{v} / \mathrm{v})$ Tween $20(\mathrm{PBST})$ at room temperature, and after washing in $1 \times$ PBST were incubated for $1 \mathrm{~h}$ at $37^{\circ} \mathrm{C}$ in a humid chamber with a mouse monoclonal antibody raised against 5-methylcytosine (anti-5-mC, Abcam AB 10805) diluted 1:200 in blocking solution. Slides were subsequently washed in $1 \times$ PBST and then incubated in Cy3-conjugated anti-mouse IgG diluted 1:100 in $1 \times \mathrm{PBS}$, $1 \% \mathrm{BSA}$ for $1 \mathrm{~h}$ at $37^{\circ} \mathrm{C}$. Finally, slides were washed in $1 \times$ PBST and mounted in antifade containing DAPI as a DNA counterstain.

\section{Image capturing}

Fluorescence signals were analysed under an epifluorescence microscope (Zeiss Axioscop2, Zeiss, Jena, Germany) with the appropriate filters. The images recorded with the Carl Zeiss Axiocam digital camera and Axiovision software (version 3.0.6.38), were separately captured and merged with Photoshop ${ }^{\circledR}$ (Adobe ${ }^{\circledR}$ Systems Inc., San Jose, California, USA). Nuclei area measurements were performed using the Axiovision measurement module 3.0.0.0 (Zeiss).

\section{Results}

Ultrasonic isolation of pollen nuclei maintains chromatin structure and integrity

A low magnitude ultrasonic treatment of fresh mature pollen in WPB buffer, and subsequent counterstaining with DAPI, revealed the binucleate structure of mature pollen of

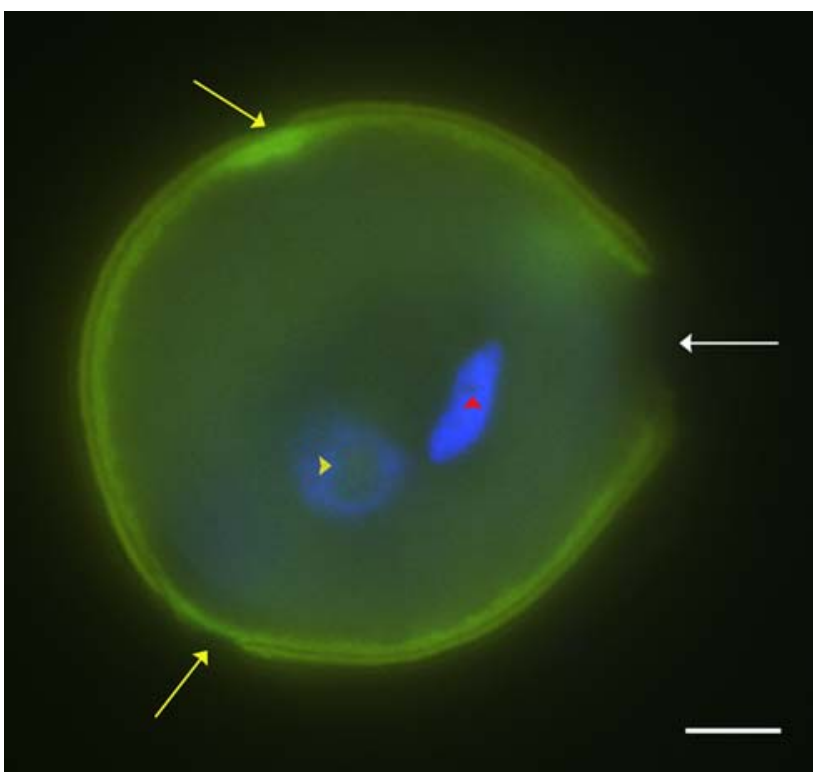

Fig. 1 Mature pollen grain of Quercus suber processed with a low magnitude ultrasonic treatment and stained with DAPI. Pollen wall is visible due to autofluorescence. Pollen grains have three apertures which were differently affected by the treatment: one aperture shows complete rupture (white arrow), while the others still remain closed (yellow arrows). The two nuclei of the bicellular pollen are differently stained by DAPI (blue signal). The yellow arrowhead indicates the large nucleolus of the vegetative nucleus and the red arrowhead, the small nucleolus of the generative nuclei. $B a r=10 \mu \mathrm{m}$

Q. suber (Fig. 1). Disruption of the exine and intine layers in one of the three cell wall apertures (white arrow, Fig. 1) allows DAPI penetration and the visualization of the two nuclei still enclosed in the pollen structure. The two nuclei could be recognized by their distinctive shape and chromatin organization: the vegetative nucleus has a nearly spherical shape. A large nucleolus detected through a faint DAPI staining (yellow arrowhead, Fig. 1), and an overall low intensity of DAPI, revealed a loose chromatin organization. The generative nucleus has an elongated shape, a small nucleolus (red arrowhead, Fig. 1) and a high intensity of DAPI staining revealing the highly condensed nature of its chromatin. Both nuclei also present marked differences in their nuclear areas, as seen in Fig. 1. A comparison between free nuclei, released from pollen grains (Fig 2a), and nuclei inside pollen grains (Fig. 1) shows that the major features of each of the two nuclear populations are maintained after ultrasonic treatment. The ratio between the areas of the vegetative and generative nuclei was also maintained both inside the pollen grains and in the free nuclei, allowing their reliable identification after immunodetection.

Nuclei of Lilium sp. were used as a control for the validation of the nuclear isolation technique in studies of nuclear organization, and in the characterization of immunodetection patterns. 
Fig. 2 Comparative distribution of methylated cytosines $(5-\mathrm{mC})$ in ultrasonic treatment- isolated vegetative and generative nuclei from Quercus suber $(\mathbf{a}, \mathbf{b}, \mathbf{c})$, and from Lillium sp. pollen grains $(\mathbf{d}, \mathbf{e}, \mathbf{f})$. DAPI staining of vegetative (left) and generative (right) nuclei (a, d). Immunodetection of 5-mC residues in both nuclei $(\mathbf{b}, \mathbf{e})$. Merged images of DAPI staining and immunodetection (d, f). Bars $=10 \mu \mathrm{m}$
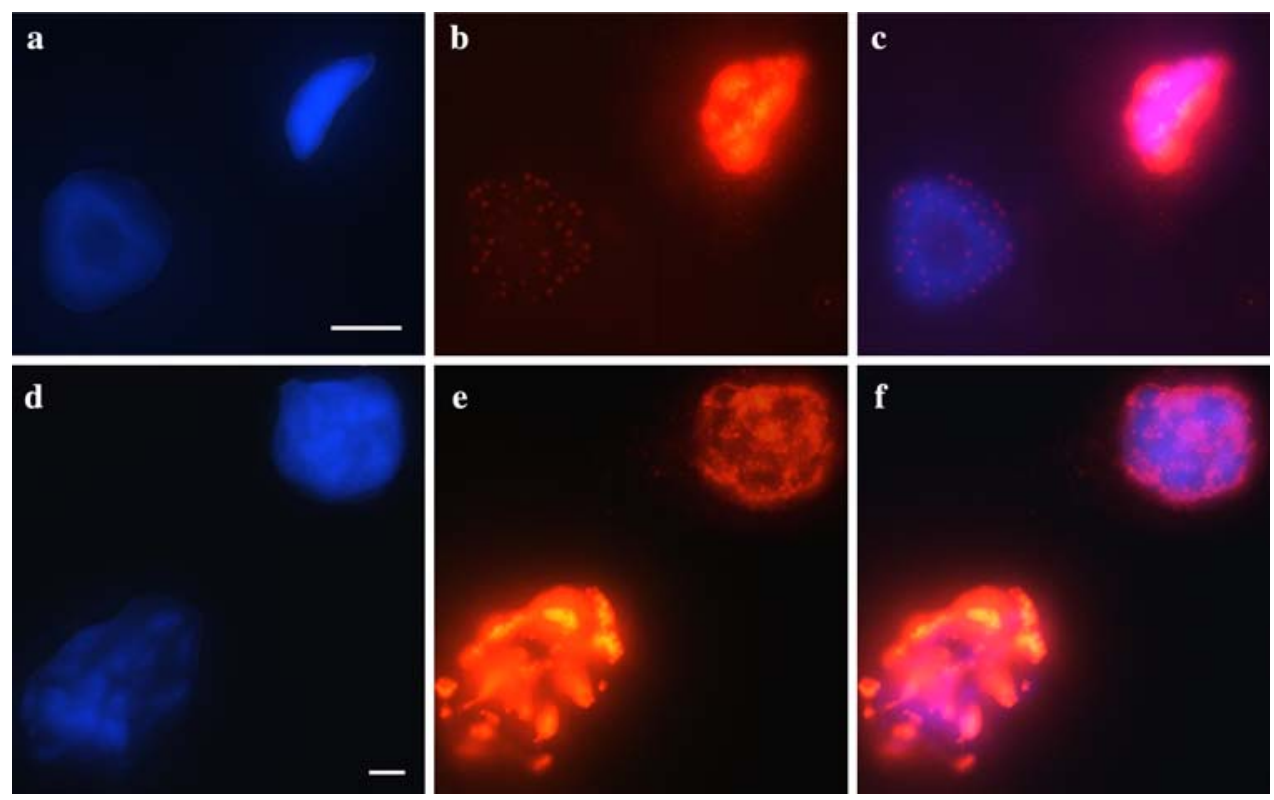

Lilium isolated nuclei have statistically significant ( $t$-test, $P<0.001$ ) larger areas (mean values for vegetative $=1,590 \pm 380 \mu \mathrm{m}^{2}, n=30$ and generative $=1,190 \pm$ $280 \mu \mathrm{m}^{2}, n=30$ ) than $Q$. suber (mean values for vegetative $195 \pm 42 \mu \mathrm{m}^{2}, n=30$ and generative nuclei $73 \mu \mathrm{m}^{2} \pm 14$, $n=30$ ). The differences can be attributed to the relative $C$ values of cork oak $(1 C=931 \mathrm{Mbp})$ and Lilium sp. Although the Lilium species used is unidentified, the DNA content data available for this genus ranges from $1 C=13,426-$ 46,942 Mbp (Bennett and Leitch 2004). The size proportion between vegetative and generative nuclei is more marked in Q. suber (mean value $0.38 \pm 0.05$ ) (Fig. 2a) than in Lilium (mean value $0.74 \pm 0.09$ ) (Fig. 2d).

Distribution patterns of 5-mC residues in vegetative and generative nuclei of Quercus suber and Lilium sp.

In addition to preserving nuclear structure and integrity the isolation of pollen by ultrasound also avoids pollen wall autofluorescence, and allows antibodies' accessibility.

In $Q$. suber the labelling pattern of the anti-5-mC antibody is highly distinctive in both the vegetative and the generative nuclei (Figs. 2b, 3). The vegetative nucleus displays a very weak label, with a discrete spotty pattern (Fig. 3) only detected when the generative nucleus is overexposed (Fig. 2b). The generative nuclei in contrast are entirely labelled, showing regions with different intensities (Fig. 3). In Lilium sp. in contrast differences in 5 -mC patterns between the two kinds of nuclei are not very marked, although the vegetative nucleus presents a stronger labelling than the generative one, (Fig. 2e) in agreement with the previous description (Janousek et al. 2000)
Distribution patterns of histones modifications in mature pollen nuclei of Quercus suber

To characterize chromatin epigenetic marks in pollen grain nuclei of $Q$. suber the following antibodies for posttranslation histone modifications were used: histone $\mathrm{H} 3$ trimethylated at lysine 4 (H3K4me3), dimethylated at lysine 9 (H3K9me2) and acetylated at lysine 9 (H3K9Ac), and histone $\mathrm{H} 4$ tetra-acetylated at lysines 5, 8, 12, 16 (H4tetraAc). Figure 3 shows that the distribution patterns of all the histones modifications studied are markedly different between the two nuclei, with the generative nuclei always more strongly labelled than the vegetative one (Table 1). All epigenetic marks studied are distributed throughout the generative nuclei, whereas in the vegetative ones they present a discrete spotty pattern with random distribution. In vegetative nuclei, H4tetraAc and $\mathrm{H} 3 \mathrm{~K} 4 \mathrm{me} 3$ isoforms show a stronger label when compared with the other isoforms (Fig. 3; Table 1) while in generative nuclei all isoforms show a very strong label except for a weaker H3K9Ac.

The lysine residue of histone $\mathrm{H} 3$ at the position nine (H3K9) can be either methylated or acetylated, and both modifications present distinct patterns for each type of nucleus. In generative nuclei the level of $\mathrm{H} 3$ acetylation is more reduced than dimethylation (Table 1), whereas in the vegetative nuclei both $\mathrm{H} 3 \mathrm{~K} 9$ modifications have similar intensities and distribution patterns (Fig. 3; Table 1).

In summary, $Q$. suber pollen nuclei show a 5 -mC pattern opposite to the one in Lilium sp. (Fig. 2). Q. suber vegetative and generative nuclei accumulate simultaneously epigenetic marks related with gene activity and gene silencing. All epigenetic marks are more represented in 


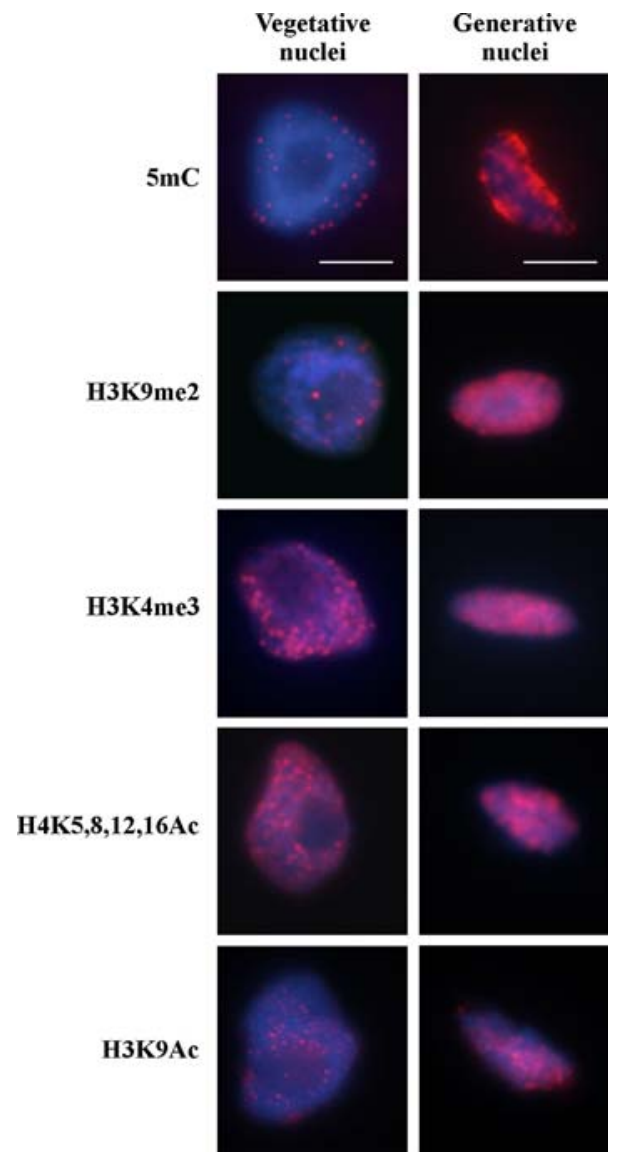

Fig. 3 Vegetative and generative pollen grain nuclei of Quercus suber. Distribution patterns of epigenetic marks associated with heterochromatin (DNA methylation, 5-mC and histone $\mathrm{H} 3$ dimethylated at lysine 9 (H3K9me2); euchromatin (histone $\mathrm{H} 3$ trimethylated at lysine 4 (H3K4me3), and acetylated isoforms of histones $\mathrm{H} 4$ (H4K5,K8,K12,K16Ac) and H3 (H3K9Ac). The exposure time in the DAPI staining and immunodetection capturing was adjusted in both vegetative and generative nuclei in order to discriminate the labelling pattern of different epigenetic marks. Bars $=10 \mu \mathrm{m}$

generative nuclei rather than in vegetatives (Fig. 3; Table 1).

\section{Discussion}

An integrated study of the epigenome of $Q$. suber, addressing the pattern of several epigenetic marks associated with gene silencing (cytosine methylation, 5-mC, and histone modifications, H3K9me) and with gene activity (histone modifications, H3K4me3, H3K9Ac and H4tetraAc) in both nuclei of the mature pollen was performed using an ultrasonic treatment for nuclei isolation.

To our knowledge this work uses ultrasonic treatment in the isolation of nuclei for in situ immunodetection assays for the first time. Our approach shows that by avoiding the fluorescence constraints of pollen grain walls, and the
Table 1 Comparative intensity of epigenetic marks between the vegetative and generative nuclei of Quercus suber mature pollen, from immunolabelling

\begin{tabular}{lll}
\hline Epigenetic marks & \multicolumn{2}{l}{ Quercus suber nuclei } \\
\cline { 2 - 3 } & Vegetative & Generative \\
\hline 5-mC & + & ++++ \\
H3K9me2 & + & ++++ \\
H3K4me3 & +++ & ++++ \\
H4K5,K8,K12,K16Ac & ++ & ++++ \\
H3K9Ac & + & ++ \\
\hline
\end{tabular}

5-mC 5-methyl cytosine, H3K9me2 histone $\mathrm{H} 3$ dimethylated at lysisne 9, H3K4me3 histone $\mathrm{H} 3$ trimethylated at lysisne 4, $H 4 K 5, K 8, K 12, K 16 A c$ histone $\mathrm{H} 4$ acetylated at lysisnes 5, 8, 12 and 16, H3K9Ac histone $\mathrm{H} 3$ acetylated at lysisne 9

Epigenetic marks label intensity visually evaluated. + Weak and scarce, ++ intermediate, +++ strong, ++++ very strong

presence of cytoplasm, not only can the two nuclear populations be perfectly recognized, but easy access of the antibodies to the chromatin also made possible the accurate characterization of distinct epigenetic marks.

Analysis of Lilium sp. pollen nuclei revealed a 5-mC distribution pattern similar to the one previously described (Janousek et al. 2000), with more intense label in the vegetative than in the generative nucleus, fully validating ultrasonic treatment for studies of chromatin organization.

The epigenetic marks studied have distinct patterns distinguishing vegetative and generative nuclei of $Q$. suber, and are different from those described in other species. Although the generative nuclei of cork oak present highly condensed chromatin they also display some features normally associated with gene activity, such as immuno signals for H4KAc and $\mathrm{H} 3 \mathrm{~K} 4 \mathrm{me} 3$, as was also observed in Lilium (Janousek et al. 2000; Okada et al. 2006). Recent studies concerning transcriptome profiling in the generative nuclei of Lilium longiflorum revealed the upregulation of 356 genes correlating epigenetic and gene expression patterns (Okada et al. 2007). The level of $\mathrm{H} 4$ acetylation has also been related with DNA replication and/or post-replication processes, such as DNA repair, maintenance of DNA methylation, and chromatin remodelling (Fuchs et al. 2006).

Comparative analysis of epigenetic marks in both nuclei of cork oak pollen revealed stronger label associated with gene silencing ( $\mathrm{H} 3 \mathrm{~K} 9 \mathrm{me} 2$ and $5-\mathrm{mC})$ in generative than in vegetative nucleus, which presents a more relaxed chromatin structure. In Lilium, a strong label of $\mathrm{H} 3 \mathrm{~K} 9 \mathrm{me} 2$ was observed in the generative nucleus (Okada et al. 2006), whereas the level of 5-mC was higher in the vegetative one (Janousek et al. 2000). In other herbaceous species studied, with either bicellular or tricellular mature pollen grains, a marked DNA methylation in the vegetative nucleus was 
also observed, although associated with a more relaxed chromatin organization compared with the generative one (Oakeley et al. 1997; Janousek et al. 2002). This apparent contradiction has been interpreted on the basis that DNA cytosine hypermethylation in the vegetative nucleus reflects temporary inactivation mechanisms acting during pollen grain maturation, which is essential for their longterm survival until they reach the stigma (Janousek et al. 2002).

In $Q$. suber the vegetative nuclei present a coherent pattern of epigenetic marks with reduced amounts of chromatin silencing marks (5-mC, and $\mathrm{H} 3 \mathrm{~K} 9 \mathrm{me})$ and high levels of active chromatin marks (H3K4me3, and H4tetraAc). This pattern contrasts with the faint or nearly absent labelling of $\mathrm{H} 3 \mathrm{~K} 4 \mathrm{me} 2$ (Okada et al. 2006) and H4KAc (Janousek et al. 2000) described for Lilium vegetative nuclei, emphasizing the differences in chromatin patterns between the herbaceous plants and the oak tree pollen grains.

We also analysed the distribution of histone $\mathrm{H} 3$ modifications at the lysine 9 (methylation, H3K9me2 and acetylation, H3K9Ac) in $Q$. suber pollen nuclei. The presence of the two types of $\mathrm{H} 3 \mathrm{~K} 9$ modifications was detected in both nuclei, although fainter signals were observed in vegetative ones. Lysine 9 of $\mathrm{H} 3$ is more methylated than acetylated in generative nuclei, whereas vegetative nuclei reveal more acetylation than methylation. While $\mathrm{H} 3 \mathrm{~K} 9 \mathrm{me} 2$ is related with gene silencing, a clear correlation between transcription and $\mathrm{H} 3 \mathrm{~K} 9 \mathrm{Ac}$ isoform has not yet been established in plants. It is known, however, that the presence of $\mathrm{H} 3 \mathrm{~K} 9 \mathrm{Ac}$ is not cell-cycle or replication dependent, occurring in both heterochromatic and euchromatic domains of Vicia faba and Hordeum vulgare, as well as in the euchromatic domains of Arabidopsis (Jasencakova et al. 2000, 2001, 2003).

Altogether our analysis of the epigenetic marks on $Q$. suber pollen grains discloses patterns that are distinct from those previously described, suggesting the existence of a correlation with different pollination/fertilization strategies in angiosperms. The chromatin compaction level of the generative nuclei is also more pronounced in $Q$. suber than in Lilium sp., which could reflect different levels of transcription between these species, relative to differences in their pollination/fertilization processes. During the progamic stage the generative nucleus needs to maintain its integrity to guarantee the proper transference of male genetic material to the female gametophyte, and it has to divide and achieve synchrony with its female counterpart which requires a long-range signalling between male and female gametophytes. For species with very long progamic phases, such as $Q$. suber, this process is even more essential to successfully achieve fertilization. We propose therefore that the complex process underlying the required synchrony between male and female gametophytes is associated with specific patterns of high order chromatin organization.

This hypothesis considers that the low level of DNA methylation associated with histone modifications characteristic of active chromatin in the $Q$. suber vegetative nucleus, indicates a high potential for transcription that can compensate the apparent chromatin silencing of the generative nucleus.

In conclusion, our data further support the idea that the epigenetic patterns associated with chromatin organization in mature nuclei of pollen grains are not universal, but reflect the fertilization timing strategies adopted by each plant species.

Acknowledgments We thank Dr. Andreas Houben (Leibniz Institute of Plant Genetics and Crop Plant Research) for kindly providing antibody against H3K9Ac. We are grateful to Augusta Barão for excellent technical assistance and Xana Castilho for her involvement with the manuscript. We also wish to thank Prof Neil Jones for his critical revision of the manuscript and editing of English. TR was supported by Fundação para a Ciência e Tecnologia with grant SFRH/ BD/13319/2003.

\section{References}

Bennett M, Leitch I (2004) Angiosperm DNA c-values database, release 5.0, Dec 2004. http://www.kew.org/cval/homepage.html

Berger SL (2007) The complex language of chromatin regulation during transcription. Nature 447:407-412

Bino RJ, Tuyl JM, De Vries JN (1990) Flow cytometric determination of relative nuclear DNA contents in bicellulate and tricellulate pollen. Ann Bot 65:3-8

Boavida LC, Varela MC, Feijó JA (1999) Sexual reproduction in the cork oak (Quercus suber L.). I. The progamic phase. Sex Plant Reprod 11:347-353

Castilho A, Neves N, Rufini-Castiglione M, Viegas W, HeslopHarrison JS (1999) 5-Methylcytosine distribution and genome organization in Triticale before and after treatment with 5-azacytidine. J Cell Sci 112:4397-4404

Craiq JM (2005) Heterochromatin-many flavours, common themes. Bioessays 27:17-28

Fuchs J, Demidov D, Houben A, Schubert I (2006) Chromosomal histone modification patterns-from conservation to diversity. Trends Plant Sci 11:199-208

Horn PJ, Peterson CL (2006) Heterochromatin assembly: a new twist on an old model. Chromosome Res 14:83-94

Houben A, Demidov D, Gernand D, Meister A, Leach CR, Schubert I (2003) Methylation of histone H3 in euchromatin of plant chromosomes depends on basic nuclear DNA content. Plant J 33:967-973

Janousek B, Zluvova J, Vyskot B (2000) Histone H4 acetylation and DNA methylation dynamics during pollen development. Protoplasma 211:116-122

Janousek B, Matsunaga S, Kejnovsky E, Zluvova J, Vyskot B (2002) DNA methylation analysis of a male reproductive organ specific gene (MROS1) during pollen development. Genome 45:930-937

Jasencakova Z, Meister A, Walter J, Turner BM, Schubert I (2000) Histone $\mathrm{H} 4$ acetylation of euchromatin and heterochromatin is cell cycle dependent and correlated with replication rather than with transcription. Plant Cell 12:2087-2100 
Jasencakova Z, Meister A, Schubert I (2001) Chromatin organization and its relation to replication and histone acetylation during the cell cycle in barley. Chromosoma 110:83-92

Jasencakova Z, Soppe WJ, Turner BM, Schubert I (2003) Histone modifications in Arabidopsis-high methylation of $\mathrm{H} 3$ lysine 9 is dispensable for constitutive heterochromatin. Plant J 33:471-480

Loureiro J, Rodriguez E, Dolezel J, Santos C (2007) Two new nuclear isolation buffers for plant DNA flow cytometry: a test with 37 species. Ann Bot 100:875-888

Oakeley J, Podesta A, Jost JP (1997) Developmental changes in DNA methylation of the two tobacco pollen nuclei during maturation. Proc Natl Acad Sci 94:11721-11725

Okada T, Singh MB, Bhalla PL (2006) Histone H3 variants in male gametic cells of lily and $\mathrm{H} 3$ methylation in mature pollen. Plant Mol Biol 62:503-512
Okada T, Singh MB, Bhalla PL (2007) Transcriptome profiling of Lilium longiflorum generative cells by cDNA microarray. Plant Cell Rep 26:1045-1052

Pan G, Zhou Y, Fowke LC, Wang H (2004) An efficient method for flow cytometric analysis of pollen and detection of $2 \mathrm{n}$ nuclei in Brassica napus pollen. Plant Cell Rep 23:196-202

Richards EJ, Elgin SC (2002) Epigenetic codes for heterochromatin formation and silencing: rounding up the usual suspects. Cell 108:489-500

Schwarzacher T, Heslop-Harrison P (2000) Practical in situ hybridization. BIOS Scientific Publishers, Oxford, p 203

Tanaka I (1997) Differentiation of generative and vegetative cells in angiosperm pollen. Sex Plant Reprod 10:1-7

Weterings K, Russell SD (2004) Experimental analysis of the fertilization process. Plant Cell 16:S107-S118 